MS relapse interval of 3.1 years. Treatment consists of IV high-dose corticosteroids, and if necessary, the addition of IV immunoglobulin and plasmapheresis, and immunosuppressive agents. Prognosis today is favorable, and contrasts with $25 \%$ mortality and $35 \%$ morbidity reported in pre-vaccination, measles-induced ADEM cases. Early use of high-dose steroids has also contributed to the improved outcome. (Menge T, Hemmer B, Nessler S et al. Acute disseminated encephalomyelitis. An update. Arch Neurol December 2005;62:1673-1680). (Olaf Stuve MD, Department of Neurology, University of Texas Southwestern Medical Center, 5323 Harry Hines Blvd, Dallas, TX 75390).

COMMENT. Although ADEM is considered a relatively rare disorder, with an estimated annual prevalence rate of $0.8 / 100,000$ of the population (Leake JA et al. Pediatr Inf Dis J 2004;23:756-764), the diagnosis is increasingly important because of the expanded usage of vaccination, the need for early steroid therapy, and the risks of permanent neurologic sequelae. The poor prognosis of patients with measles-related ADEM should be a warning to parents who are persuaded to forego immunization of a child because of unsubstantiated reports of potential risks of autism.

Patients with onset of ADEM before 5 years of age have a lower mean intelligence quotient $(\mathrm{p}<0.01)$, significantly lower scores on reading and spelling tests $(\mathrm{p}<0.001)$, and a higher incidence of severe behavioral and emotional problems, compared to controls of the same age and socioeconomic status (Jacobs RK et al. Pediatr Neurol 2004;31:191-197). This study finds standard scores on spelling significantly lower in a young onset group compared to older onset patients with ADEM $(\mathrm{p}<0.05)$. Long-term neuropsychological dysfunction may occur in children who develop ADEM in early childhood, despite the absence of persisting neurologic deficits. (see Ped Neur Briefs 2004;18:66).

\title{
KLEINE-LEVIN SYNDROME
}

A systematic review of all published cases of Kleine-Levin syndrome (KLS) described in 195 articles since 1962 is presented from Stanford University Center for Narcolepsy, Palo Alto, CA; the Kleine-Levin Syndrome Foundation, Boston, MA; and Hopital Pitie-Salpetriere, Paris, France. KLS is primary or infrequently, secondary to stroke, brain trauma and other disorders. Of 168 primary cases, $68 \%$ occurred in males, and the median age of onset was 15 years (range 4-82 years). Onset was preceded by infection in $43 \%$ of cases, commonly a nonspecific fever or flu-like illness, upper respiratory tract infection or tonsillitis, and less often, gastroenteritis, Epstein-Barr virus, varicella-zoster virus, Asian influenza, and streptococcus. Hypersomnia is the characteristic symptom, occurring in $100 \%$ cases. Other common symptoms include mental disorders (confusion, amnesia, derealization, hallucinations) in $98 \%$, eating disorders $(80 \%)$, hypersexuality (masturbation, inappropriate sexual advances) (43\%), compulsions (29\%), and depression $(48 \%)$. Episodes of hypersomnia lasted 10 days, recurred every 3.5 months, and recovery followed in 8 years. Women had a longer disease course than males $(9+/-8.7$ years vs $5.4+/-$ 5.6 years, $\mathrm{p}=0.01$ ). Treatment with stimulants (mainly amphetamines) relieved somnolence in $40 \%$. Lithium prevented relapses in $41 \%$ cases cf to $19 \%$ with no relapse in untreated cases $(p=0.02)$. CSF was normal. EEG showed only nonspecific diffuse slowing in $70 \%$ patients.

Brain CT and MRI were normal. Hormonal tests showed no consistent abnormality. In 18 
patients with secondary KLS, 5 had stroke or brain trauma, and the remainder had various diseases, including genetic, infectious encephalitis, and multiple sclerosis. Symptoms in secondary cases began later than in primary KLS, and the disease course was longer and more severe. (Arnulf I, Zeitzer JM, File J et al. Kleine-Levin syndrome: a systematic review of 186 cases in the literature. Brain December 2005;128:2763-2776). (Respond: Dr I Arnulf, Federation des pathologie du Sommeil, Hopital Pitie-Salpetriere, 47-83 Boulevard de l'Hopital, 75013 Paris, France).

COMMENT. First named KLS by Critchley et al (1942), these authors emphasized the male preponderance, adolescent onset of episodic hypersomnia, compulsive eating, and spontaneous recovery. The infectious factor in causation of KLS requires further study, especially in relation to viral or post-infectious autoimmune encephalitis, with involvement of the hypothalamus. Neuropathology in 2 primary KLS cases showed inflammatory changes in the thalamus, hypothalamus, and basal ganglia. A genetic factor is suggested, since onesixth of patients were Jewish. Epilepsy and depressive causes have been ruled out.

\section{CORTICOSTEROIDS IN HERPES SIMPLEX VIRUS ENCEPHALITIS}

A non-randomized retrospective study of 45 adult patients (12 young adults) with herpes simplex virus encephalitis (HSVE), treated with acyclovir alone (23 patients) and acyclovir plus corticosteroids (22 patients), was conducted at Nihon University School of Medicine, Tokyo, Japan. At 3 months, a poor outcome was found in older patients, those with lower Glasgow Coma Scores at initiation of therapy, and in those not receiving corticosteroids in the acute stage. (Kamei S, Sekizawa T, Shiota $\mathrm{H}$ et al. J Neurol Neurosurg Psychiatry Nov 2005;76:1544-1549). (Respond: Dr S Kamei, Division of Neurology, Department of Medicine, Nihon University School of Medicine, 30-1 Oyaguchikamimachi, Itabashi-ku, Tokyo 173-8610, Japan).

COMMENT. This study suggests that a combination of acyclovir with corticosteroids initiated at the acute stage of HSVE is a predictor of a better prognosis than acyclovir alone. The beneficial effects of corticosteroids may be explained by reduction of brain edema and regulation of the host immune response, decreasing the extent of viral infection.

Openshaw $\mathrm{H}$ and Cantin EM, in an editorial commentary ( $\mathbf{J}$ Neurol Neurosurg Psychiatry Nov 2005;76:1469), opine that the effectiveness of coriicosteroids in HSVE can only be determined by prospective, randomized studies. The benefits observed in the above retrospective study could be related to selection of cases and to the corticosteroid therapy per se.

Concerns that early corticosteroid administration may increase viral CNS spread, leading to a restriction of steroids to cases with significant brain edema, may not be justified, based on recent animal studies cited by Kamei et al (Thompson KA et al. Herpes simplex replication and dissemination is not increased by corticosteroid treatment in a rat model of focal herpes encephalitis. J Neurovirol 2000;6:25-32; Meyding-Lamade UK et al. Experimental HSVE: a combination therapy of acyclovir and glucocorticoids reduces longterm MRI abnormalities. J Neurovirol 2003;9:118-125). Early diagnosis is a factor in prognosis, and a characteristic EEG multifocal periodic pattern may be helpful when CT and ultrasound are normal in neonatal HSVE (Mikati MA et al. Neurology 1990;40:1433-37). 